\title{
FAT1 mutations cause a glomerulotubular nephropathy
}

Heon Yung Gee ${ }^{1,2, \star}$, Carolin E. Sadowski ${ }^{1, \star}$, Pardeep K. Aggarwal ${ }^{3}$, Jonathan D. Porath ${ }^{1}$, Toma A. Yakulov ${ }^{4}$, Markus Schueler $^{1}$, Svjetlana Lovric ${ }^{1}$, Shazia Ashraf ${ }^{1}$, Daniela A. Braun ${ }^{1}$, Jan Halbritter ${ }^{1}$, Humphrey Fang ${ }^{1}$, Rannar Airik1, Virginia Vega-Warner ${ }^{5}$, Kyeong Jee Cho ${ }^{2}$, Timothy A. Chan ${ }^{6}$, Luc G.T. Morris ${ }^{6}$, Charles ffrench-Constant ${ }^{7}$, Nicholas Allen ${ }^{8}$, Helen McNeill ${ }^{9}$, Rainer Büscher ${ }^{10}$, Henriette Kyrieleis ${ }^{11}$, Michael Wallot ${ }^{11}$, Ariana Gaspert ${ }^{12}$, Thomas Kistler ${ }^{13}$, David V. Milford ${ }^{14}$, Moin A. Saleem ${ }^{15}$, Wee Teik Keng ${ }^{16}$, Stephen I. Alexander ${ }^{17}$, Rudolph P. Valentini ${ }^{18}$, Christoph Licht ${ }^{19}$, Jun C. Teh ${ }^{19}$, Radovan Bogdanovic ${ }^{20}$, Ania Koziell21, Agnieszka Bierzynska ${ }^{15}$, Neveen A. Soliman²2,23, Edgar A. Otto ${ }^{5}$, Richard P. Lifton ${ }^{24,25}$, Lawrence B. Holzman ${ }^{26}$, Nicholas E. S. Sibinga ${ }^{27}$, Gerd Walz ${ }^{4}$, Alda Tufro ${ }^{3}$ \& Friedhelm Hildebrandt ${ }^{1,25}$

Steroid-resistant nephrotic syndrome (SRNS) causes 15\% of chronic kidney disease (CKD). Here we show that recessive mutations in FAT1 cause a distinct renal disease entity in four families with a combination of SRNS, tubular ectasia, haematuria and facultative neurological involvement. Loss of FAT1 results in decreased cell adhesion and migration in fibroblasts and podocytes and the decreased migration is partially reversed by a RAC1/CDC42 activator. Podocyte-specific deletion of Fat1 in mice induces abnormal glomerular filtration barrier development, leading to podocyte foot process effacement. Knockdown of Fat1 in renal tubular cells reduces migration, decreases active RAC1 and CDC42, and induces defects in lumen formation. Knockdown of fat 1 in zebrafish causes pronephric cysts, which is partially rescued by RAC1/CDC42 activators, confirming a role of the two small GTPases in the pathogenesis. These findings provide new insights into the pathogenesis of SRNS and tubulopathy, linking FAT1 and RAC1/CDC42 to podocyte and tubular cell function.

\footnotetext{
${ }^{1}$ Division of Nephrology, Boston Children's Hospital, Harvard Medical School, Boston, Massachusetts 02115, USA. ${ }^{2}$ Department of Pharmacology, Brain Korea 21 PLUS Project for Medical Sciences, Yonsei University College of Medicine, Seoul 03722, Korea. ${ }^{3}$ Department of Pediatrics, Yale University School of Medicine, New Haven, Connecticut 06520, USA. ${ }^{4}$ University Freiburg Medical Center, Freiburg 79106, Germany. ${ }^{5}$ Department of Pediatrics, University of Michigan, Ann Arbor, Michigan 48109, USA. ${ }^{6}$ Human Oncology and Pathogenesis Program, Memorial Sloan Kettering Cancer Center, New York, New York 10065, USA. ${ }^{7}$ MRC Centre for Regenerative Medicine, Multiple Sclerosis Society Centre for Translational Research, University of Edinburgh, Edinburgh EH16 4UU, UK. ${ }^{8}$ School of Biosciences, Cardiff University, Museum Avenue, Cardiff CF10 3 AX, UK. ${ }^{9}$ Department of

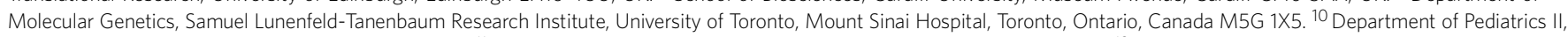
University Hospital of Essen, Essen 45147, Germany. ${ }^{11}$ Department of Pediatrics, Bethanien Hospital, Moers 47441, Germany. ${ }^{12}$ Institute of Surgical Pathology, University Hospital

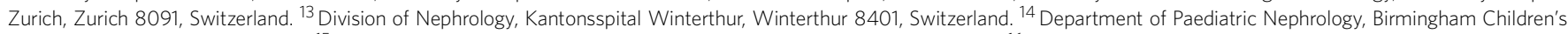
Hospital, Birmingham B4 6NH, UK. ${ }^{15}$ Children's and Academic Renal Unit, University of Bristol, Bristol BS1 5NB, UK. ${ }^{16}$ Department of Genetics, Hospital Kuala Lumpur, Kuala Lumpur

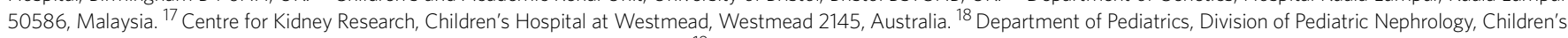
Hospital of Michigan/Wayne State University, Detroit, Michigan 48201, USA. ${ }^{19}$ Division of Nephrology, The Hospital for Sick Children and University of Toronto, Toronto, Ontario, Canada M5G 1X8. ${ }^{20}$ Institute for Mother and Child Health Care of Serbia "Dr Vukan Čupić", Department of Nephrology, University of Belgrade, Faculty of Medicine, Belgrade 11000, Serbia. ${ }^{21}$ Department of Experimental Immunobiology, Division of Transplantation Immunology \& Mucosal Biology, King's College London, Faculty of Life Sciences \& Medicine, 5th floor Tower Wing, Guy's Hospital, Great Maze Pond, London SE1 9RT, UK. 22 Department of Pediatrics, Center of Pediatric Nephrology \& Transplantation, Kasr Al Ainy School of Medicine, Cairo University, Cairo 11562, Egypt. ${ }^{23}$ Egyptian Group for Orphan Renal Diseases, Cairo 11562, Egypt. ${ }^{24}$ Department of Genetics, Yale University School of Medicine, New Haven, Connecticut 06520, USA. ${ }^{25}$ Howard Hughes Medical Institute, Chevy Chase, Maryland 20815, USA. ${ }^{26}$ Renal-Electrolyte and Hypertension Division, Perelman School of Medicine, University of Pennsylvania, Philadelphia, Pennsylvania 19104, USA. ${ }^{27}$ Wilf Family Cardiovascular Research Institute and Department of Medicine/Cardiology, Albert Einstein College of Medicine, Bronx, New York 10461, USA. * These authors contributed equally to this work. Correspondence and requests for materials should be addressed to A.T. (email: alda.tufro@yale.edu) or to F.H. (email: Friedhelm.Hildebrandt@childrens.harvard.edu).
} 
$\mathrm{N}$ ephrotic syndrome (NS) results in proteinuria, hypoalbuminemia and oedema, and is classified by its response to steroid treatment into steroid-sensitive NS and steroid-resistant NS (SRNS). SRNS leads to chronic kidney disease (CKD) within a few years of onset, requiring renal replacement therapy for survival. It causes $15 \%$ of all end-stage kidney disease that manifests by 25 years of age ${ }^{1}$. SRNS is considered one of the most intractable kidney diseases, because it has a $30 \%$ risk of recurrence after renal transplant if SRNS is idiopathic. However, in SRNS with a monogenic cause the risk of recurrence is reduced from 35 to $8 \%$ (ref. 2). Histologically, SRNS manifests as 'focal segmental glomerulosclerosis' (FSGS) or as the early-onset developmental variant 'diffuse mesangial sclerosis' $(\mathrm{DMS})^{3}$. The first insights into the pathogenesis of SRNS were gained by the discovery of single-gene (monogenic) causes of SRNS, revealing that the encoded proteins are essential for the function of the glomerular podocyte ${ }^{4,5}$. Podocytes are neuron-like cells that extend multiple tubulin-based primary processes that branch into secondary actin-based foot processes. Foot processes interdigitate with those of neighbouring podocytes, forming between them the glomerular slit diaphragm, which is critical for the filtering process. Loss of foot process and slit membrane integrity causes glomerular protein leakage and SRNS.

Advances in molecular diagnostics enables identification of the primary cause of disease in a large fraction of individuals with SRNS. In a worldwide cohort of 1,783 families, a monogenic cause of SRNS was detected in 1 of 27 genes in $\sim 30 \%$ of SRNS cases manifesting before age 25 years ${ }^{6}$. Gene identification also enables disease modelling in cell-based and animal models of gene knockdown or knockout, facilitates screening for small molecule therapeutics and permits an aetiologic classification of SRNS for therapeutic trials.

Identification of monogenic causes of SRNS has revealed that the encoded proteins are part of protein-protein interaction complexes that functionally participate in distinct cellular tasks and signalling pathways within podocytes, including:

(i) Podocyte slit membrane signalling (NPHS1, NPHS2, CD2AP, PLCE1) $)^{7-10}$,

(ii) Actin-binding complexes within the foot process actin network (ACTN4, INF2, MYO1E and ANLN) ${ }^{11-14}$,

(iii) Actin regulation by $\mathrm{RHO} / \mathrm{RAC} / \mathrm{CDC} 42$ (ARHGAP24, ARHGDIA, KANK1 and 2) ${ }^{15-17}$,

(iv) Integrin/laminin signalling in podocyte focal adhesions at the basement membrane (LAMB2 and ITGA3) ${ }^{18-21}$,

(v) Coenzyme $\mathrm{Q}_{10}$ biosynthesis components (COQ2, COQ6, PDSS2 and ADCK4) ${ }^{22-25}$,

(vi) Transcription factors expressed in podocytes (WT1 and SMARCAL1) $)^{21,26}$, as well as

(vii) Calcium signalling (TRPC6) $)^{27,28}$ and caveolin signalling $(\mathrm{EMP} 2)^{29}$.

Although disease mechanisms of SRNS are not well-understood, altered podocyte cell-migration rate represents a relevant assay of pathogenicity for most SRNS disease-causing alleles ${ }^{16,30}$. Actin remodelling by members of the 'RHO family of small GTPases' RHOA, RAC1 and CDC42 (henceforth 'RHO GTPases') regulates podocyte cell-migration rate ${ }^{30}$.

Because genetic mapping data indicate a multitude of additional loci-bearing mutations that might cause monogenic forms of SRNS, here we perform homozygosity mapping (HM) and whole-exome sequencing to identify novel monogenic SRNS genes. We identify mutations in FAT1 that cause a human glomerulotubular disease with features of both SRNS and tubular ectasia. Knockdown of Fat1 in renal tubular epithelial cells decreases cell-cell adhesion, cell migration and activity of small RHO-like GTPases RAC1 and CDC42, but also induces defects in the formation of the tubular cell lumen. Podocyte-specific Fat1 loss-of-function recapitulates the NS phenotype in a mouse model. Furthermore, knockdown of fat1 in zebrafish causes formation of pronephric cysts, which is partially rescued by RAC1/CDC42 activators, confirming a role of the two small GTPases in the pathogenesis of this renal disease.

Identification of this new glomerulotubular disease entity demonstrates that FAT1 is necessary for glomerular as well as tubular function. These findings provide new insights into the pathogenesis and therapeutic approaches for SRNS and tubular ectasia, and link FAT1 and RAC1/CDC42 to podocyte and tubular cell function.

\section{Results}

FAT1 mutations cause a glomerulotubular nephropathy. We previously showed that a causal mutation in 1 of 27 different single genes can be identified in $\sim 30 \%$ of cases with SRNS that manifest before 25 years of age $\mathrm{e}^{31}$. However, genetic mapping data strongly suggest a multitude of additional monogenic causes of SRNS $^{32}$. The finding that mutations in identified monogenic SRNS genes are very rare (LAMB2 (refs 19,33), PLCE1 (ref. 10) and COQ6 (ref. 23) highlights the need to identify additional single-gene causes of SRNS in single affected families. To overcome this limitation, we combined whole exome sequencing (WES) with $\mathrm{HM}^{32,34-36}$.

A4623, a Turkish boy from consanguineous parents, was diagnosed with intellectual disability, pulmonary artery stenosis and bilateral blepharoptosis in early childhood. Magnetic resonance imaging showed pachygyria and Virchow-Robin spaces. He was admitted to the hospital at the age of 15 years because of proteinuria and haematuria. Kidney biopsy showed a glomerulotubular nephropathy. Typical features of SRNS, including glomerular podocyte foot process effacement (Fig. 1a), were seen together with tubular dilation, tubulointerstitial infiltrations and irregular tubular basement membranes (Fig. 1b). We performed HM in A4623. HM yielded 13 regions of homozygosity by descent that represents candidate regions for a recessive disease gene (Fig. 1g) (ref. 32). Following WES and filtering of variants from normal reference sequence, three rare variants in FAT1, PIDD and DZIP1 remained (Supplementary Table 1) in A4623. Mutations in the 27 known SRNS genes were excluded by evaluation of the WES data. The variant in the FAT1 gene, which encodes the FAT atypical cadherin 1 , is a homozygous protein truncating mutation (p.P1032Cfs ${ }^{\star} 11$ ) (Table 1, Fig. 1h-j and Supplementary Table 1).

One child of Arab origin (A3027) presented with proteinuria and haematuria, and renal ultrasound showed increased echogenicity (Supplementary Fig. 1). A3027 was diagnosed with Ewing sarcoma, and lung and spinal metastasis at the age of 15 years. He rapidly progressed to renal failure and despite radiotherapy, chemotherapy and haemodialysis he died at the age of 19 years. Because of the rapid onset of renal failure, kidney biopsy was not performed but ultrasound showed echogenicity and urine analysis showed haematuria and proteinuria. There was no other affected individual in the family. HM of this individual yielded segments of homozygosity by descent with a cumulative genomic length of $215 \mathrm{Mb}$ (Supplementary Table 1). Following variant filtering by $\mathrm{HM}$ and WES, two rare missense variants in FAT1 and EHD1 remained (Supplementary Table 1). Mutations in the 27 known SRNS genes were excluded by evaluation of the WES data. The variant (c.857A > F;p.N286S) in FAT1 is reported as a SNP in the dbSNP database, however, its minor allele frequency is 0.0002 and it never occurred in the homozygous state (Table 1 and Fig. $1 \mathrm{~h}-\mathrm{j}$ ). The FAT1 variant alters an amino-acid residue conserved throughout evolution down to Drosophila melanogaster (Table 1). 

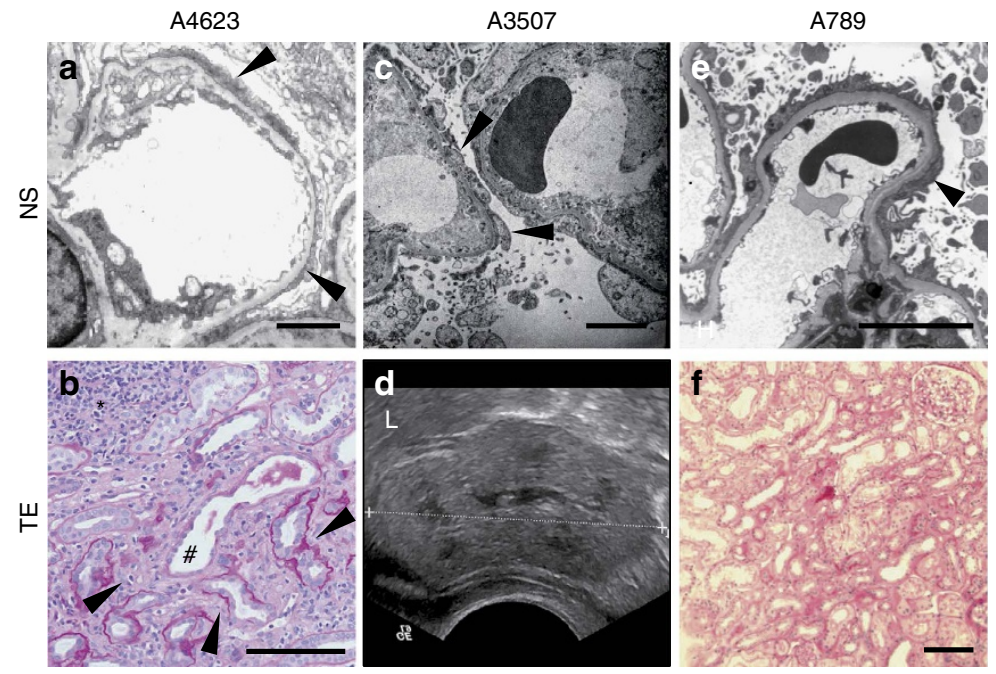

g
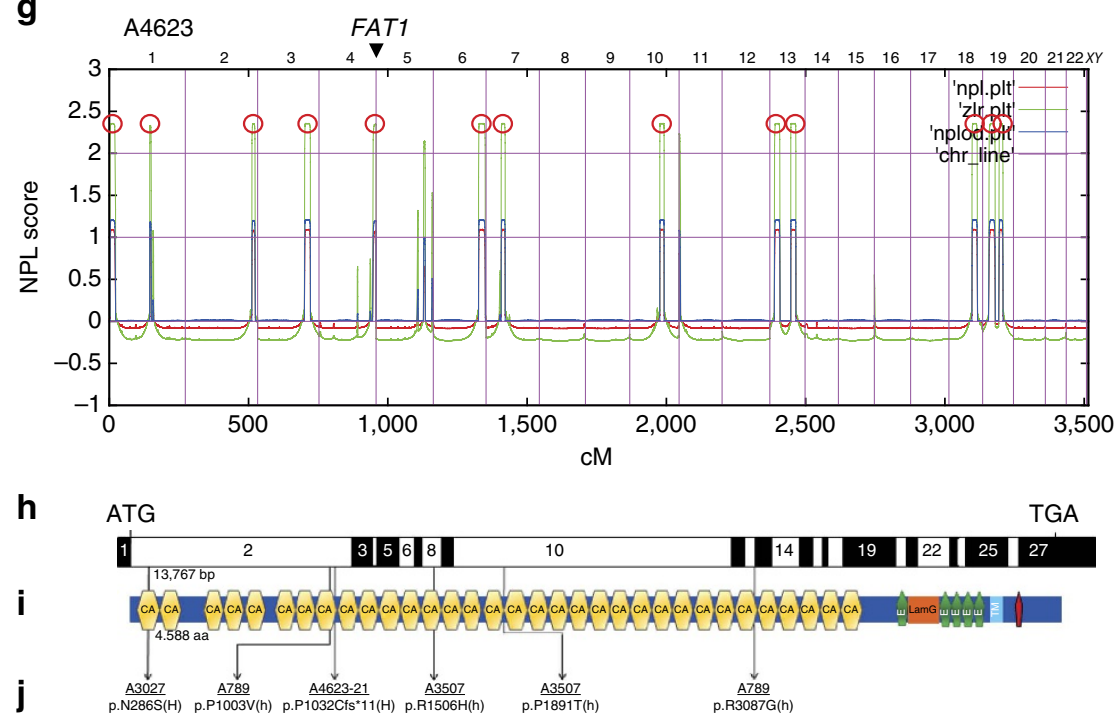

Figure 1 | FAT1 mutations cause a glomerulotubular nephropathy. (a) Electron microscopy in A4623 with FAT1 mutation demonstrates the nephrotic syndrome (NS) feature of foot process effacement (arrowheads). Scale bar, $5 \mu \mathrm{m}$. (b) Renal histology of individual A4623 exhibits cystic dilation of renal tubules (hash), interstitial infiltrations (asterisks), and tubular basement membrane disruptions (arrowheads). Scale bar, $100 \mu \mathrm{m}$. (c) In A3507 with FAT1 mutation electron microscopy reveals the NS feature of extensive foot process effacement with microvilli formation (arrowheads). Scale bar, $5 \mu$ m.

(d) Renal ultrasound of individual A3507 demonstrates loss of corticomedullary differentiation and increased echogenicity (L, liver). (e) Electron microscopy of A789 shows foot process effacement (arrowheads). Scale bar, $5 \mu \mathrm{m}$. (f) Renal histology of A789 shows tubulointerstitial infiltrates. Scale bar, $100 \mu \mathrm{m}$. (g) Homozygosity mapping identified 13 recessive candidate loci in individual A4623 with NS and tubular ectasia (TE). Non-parametric lod scores (NPL) were calculated and plotted across the human genome. The $x$-axis shows Affymetrix $250 \mathrm{~K}$ Styl array SNP positions on human chromosomes concatenated from $p$-ter (left) to $q$-ter (right). Genetic distance is given in cM. 13 maximum NPL peaks (red circles) indicate candidate regions of homozygosity by descent. The FAT1 locus (arrowhead) is positioned within the maximum NPL peak on chromosome 4q. (h) Exon structure of human FAT1 cDNA. FAT1 contains 27 exons. Positions of start codon (ATG) and of stop codon (TGA) are indicated. (i) Domain structure of FAT1. Protein domains are depicted by coloured bars in relation to encoding exon positions (h). FAT1 contains 33 cadherin domains (CA), a laminin G domain (LamG) and five epidermal growth factor (EGF)-like repeat domains (green bullets) in its extracellular region, followed by a transmembrane region (light blue bar) and a C-terminal cytoplasmic domain containing a PTB-like motif (red bar) with a PDZ-binding motif (-HTEV). (j) Two homozygous (H) and four different compound-heterozygous FAT1 mutations (h) detected in four families with a glomerulotubular nephropathy. Family numbers (underlined), mutations and predicted translational changes are indicated (see also Table 1).

When performing highly parallel sequencing ${ }^{37}$ of all FAT1 exons in 1,500 additional individuals with features of NS and 800 individuals with features of tubulointerstitial nephroapathy, we detected in 2 additional families 4 different recessive (biallelic) mutations of FAT1 (Table 1, Fig. 1j). In a female African-American girl (A789) from non-consanguineous parents, another compound-heterozygous mutation was detected (c.3008C > T, p.A1003V and c.9259C > G, p. R3087G). At the age of 9 years, kidney biopsy revealed minimal change NS.
Urine analysis showed haematuria and proteinuria. After steroid resistance, cyclosporine A was started. One year after onset of NS, she was diagnosed with Morbus Hodgkin and chemotherapy was performed. Further information was not available and segregation analysis was not performed since the girl was lost for follow-up. There was no other affected individual in the two families. A3507, an African girl from non-consanguineous parents, showed haematuria and proteinuria at the age of 2 years. Kidney biopsy revealed DMS at the age of 2 years and she received unilateral 


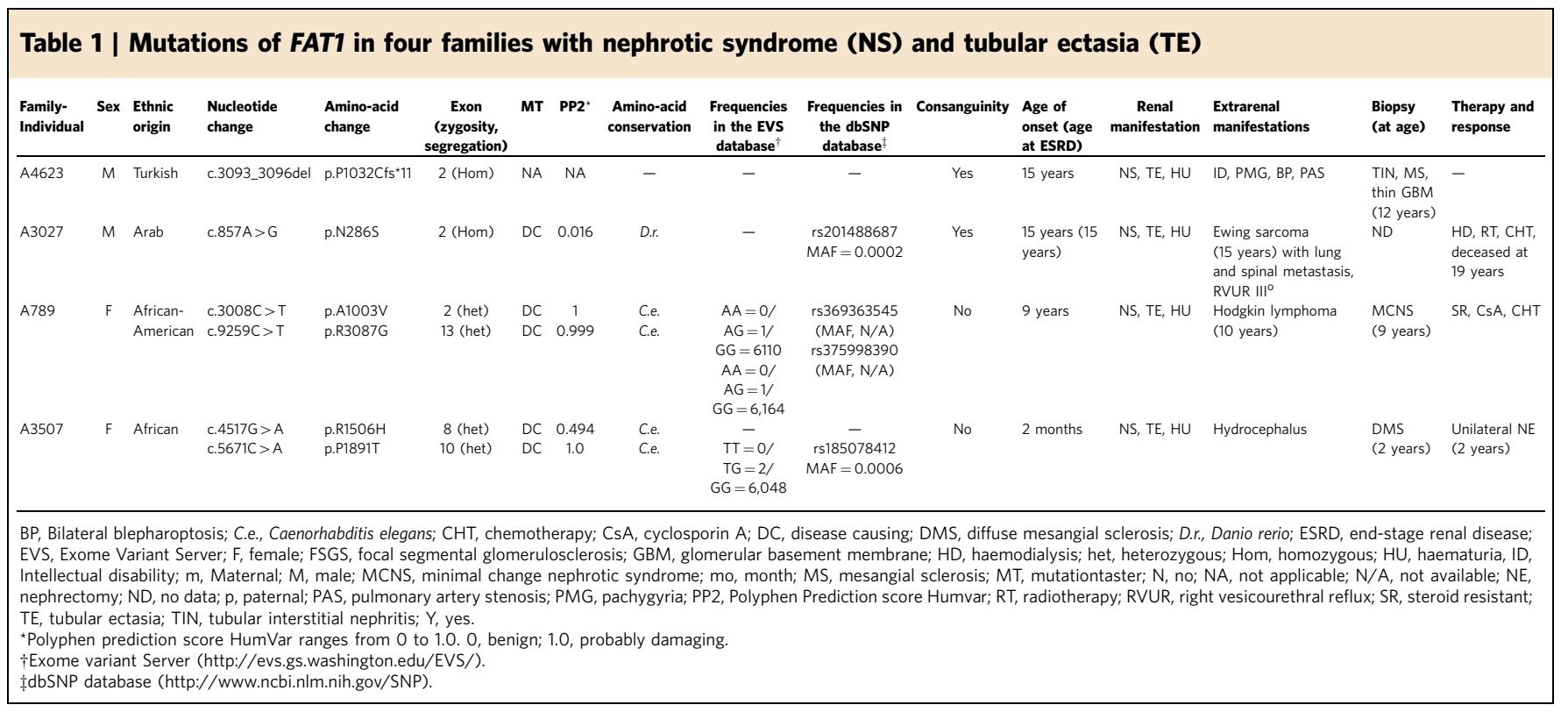

nephrectomy at the age of 2 years. In addition, a mild hydrocephalus was drained with a ventriculoperitoneal shunt. One of the compound-heterozygous mutation (c.4517G $>A$, p.R1506H) was detected in her mother, but not the other. DNA from the father was not available.

Three out of four mutations are reported as a SNP in the dbSNP database, but their allele frequencies are either not available or very rare (Table 1). For exclusion of known genetic causes of SRNS, 27 known genes previously linked to SRNS were screened in these individuals, but no explanatory mutations were detected ${ }^{6}$. Interestingly, all affected individuals exhibited a glomerulotubular nephropathy of SRNS, tubular ectasia and microscopic haematuria (Fig. 1a-f). In addition, two individuals had a central nervous system phenotype, including hydrocephalus and pachygyria (with intellectual disability and bilateral blepharoptosis) (Table 1, Supplementary Fig. 1).

FAT1 is a member of a small family of vertebrate cadherin-like genes, designated FAT1-FAT4 in humans, whose orthologues were first recognized in Drosophila ${ }^{38}$. The FAT1 protein contains 33 cadherin repeats, followed by 5 epidermal growth factor (EGF)-like repeat domains, a laminin G domain, a transmembrane domain and an intracellular domain (Fig. 1i).

FAT cadherins play a role in cell migration, lamellipodia dynamics, cell polarity and cell-cell adhesion. Fat cadherins have been reported to interact with Ena/VASP proteins, atrophins, $\beta$-catenin, scribble and HOMER1-HOMER3, thereby influencing Wnt and Hippo signalling and the regulation of planar cell polarity (PCP), the process by which cells become polarized and organized within the plane of an epithelial sheet. The Fat1 ${ }^{-/-}$ mouse displays abnormal podocyte foot processes, brain developmental defects and eye abnormalities ${ }^{39}$.

FAT1 defects reduce cell migration and cell-cell adhesion. To examine if FAT1 mutations cause defects of cellular function, we obtained fibroblasts from individual A4623. By immunoblotting of patient fibroblast cellular lysates (Fig. 2a) using an antibody that recognizes the intracellular domain of FAT1 (ref. 40) (Fig. 1i) we demonstrated absence of FAT1 protein in fibroblasts of individual A4623 (Fig. 2a), who carries a homozygous truncating mutation of FAT1 (p.P1032Cfs ${ }^{\star} 11$ ) (Table 1, Fig. 1j).
To further characterize cellular defects in patient fibroblasts, we employed a cell-migration assay (Fig. 2b), which has been previously used to demonstrate deficiencies of cell migration rate in models of multiple monogenic SRNS genes, including ARHGDIA ${ }^{16}$, KANK2 (ref. 17), MYO1E ${ }^{13,41}$, ARHGAP24 (ref. 15) and FAT1 (refs 40,42). We found that fibroblasts from individual A4623 with a homozygous truncating mutation of FAT1 p.P1032Cfs ${ }^{\star} 11$ exhibited decreased migration rate (Fig. 2b,c). The decreased migration of fibroblasts from A4623 with FAT1 mutation was partially rescued by RAC/CDC42 activator II, indicating that RHO-like small GTPase activity may be relevant to the pathogenesis of the disease caused by loss of FAT1 (Fig. 2b,c). Similarly, knockdown of FAT1 in differentiated cultured podocytes led to decreased migration rate (Fig. 2d,e and Supplementary Fig. 2). This effect was likewise mediated by RHO GTPases, because decreased migration was partially mitigated by treatment with RAC/CDC42 activator II (Fig. 2d,e). To confirm whether knockdown of FAT1 affects RHO GTPases, we used GST-rhotekin to assay the active GTP-bound state of RHOA and used GST-PAK1 to assay the active states of RAC1 and CDC42. Knockdown of FAT1 in differentiated podocytes decreased the active state of RAC1 and CDC42 (Supplementary Fig. 3a,c,d) but had no effect on RHOA (Supplementary Fig. 3b,e), indicating RAC1 and CDC42 are more relevant to the pathogenesis of SRNS caused by loss of FAT1. In addition, overexpression of dominant negative CDC42 (T17N) decreased cell migration in control differentiated cultured podocytes, but failed to further decrease migration in podocytes with FAT1 knockdown, suggesting that CDC42 is a downstream mediator of FAT1-mediated cell migration (Supplementary Fig. 4).

We then tested whether there was decreased cell-cell adhesion, which was recognized previously as a feature of FAT1 loss-offunction ${ }^{43}$. We found that fibroblasts from individual A4623 with the homozygous truncating mutation exhibited decreased cell-cell adhesion (Fig. 2f). Moreover, specific knockdown of FAT1 in differentiated cultured podocytes also impaired cell-cell adhesion, confirming that this effect is due to loss of FAT1 expression (Fig. 2g).

Podocyte-specific Fat1 loss-of-function leads to FSGS. In addition to in vitro cell-based studies, we examined the effect of 
a

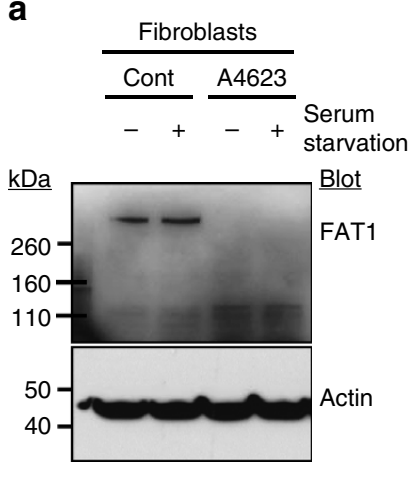

b

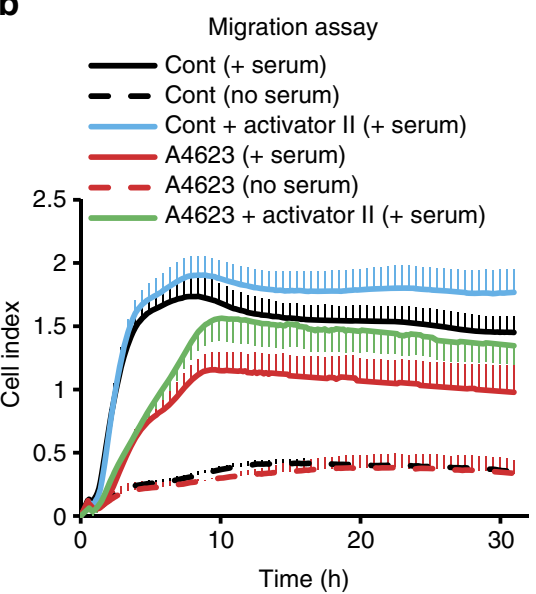

c

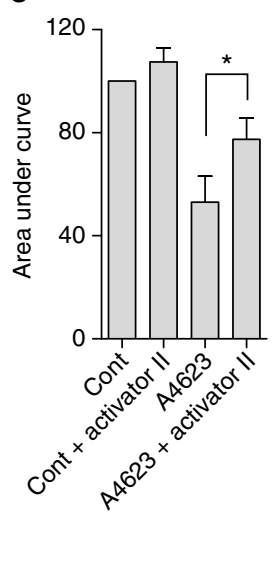

d

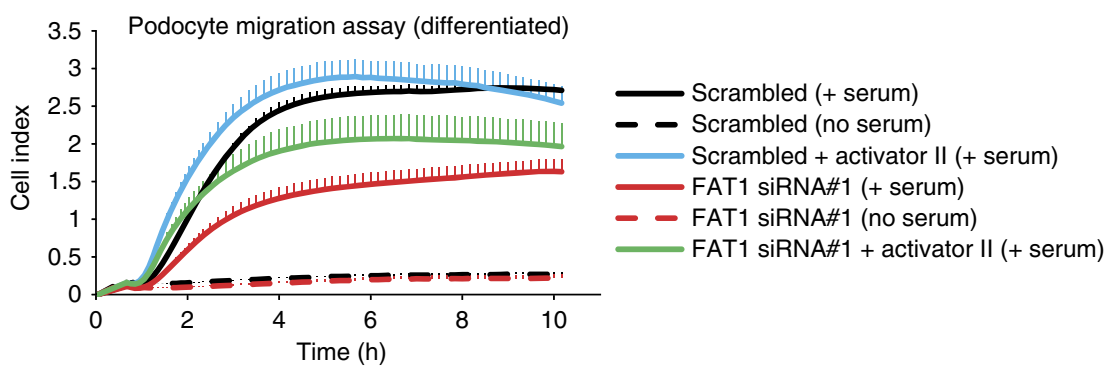

e

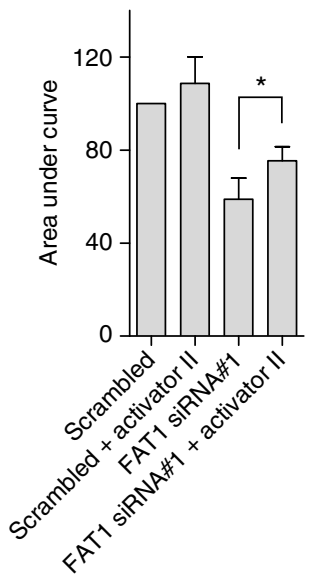

f

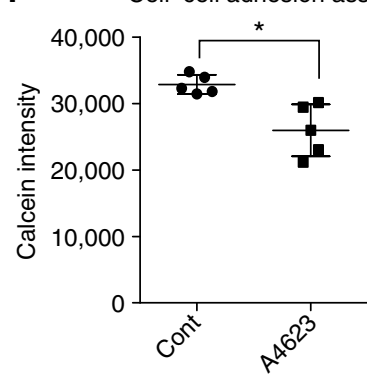

Cell-cell adhesion assay (differentiated podocytes)

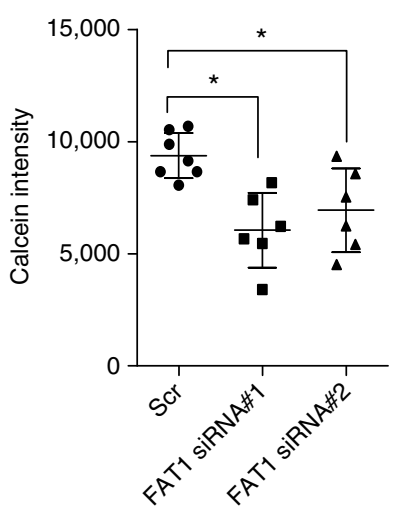

Figure 2 | Loss of FAT1 causes defects in migration and cell-cell adhesion in fibroblasts and cultured podocytes. (a) Cell lysates from fibroblasts of individuals A4623 were collected and protein level of FAT1 was analysed by western blotting with FAT1-GST antibody. FAT1-GST antibody recognizes the intracellular domain of FAT1 (C-terminal 385 aa of mouse FAT1), thus demonstrating the deficiency of the truncated FAT1 protein (p.P1032Cfs ${ }^{\star} 11$ ) resulting from c.3093_3096del FAT1 mutation in A4623. (b) Cell migration assay using the xCELLigence system. Fibroblasts from A4623 show decreased migration compared with control. Note that the decrease in migration of A4623 fibroblasts was partially rescued by the RAC/CDC42 activator II. Each cell index value corresponds to the average of more than triplicates and s.d. is in only one direction for clarity. (c) Bar graphs represent the area under curves of $\mathbf{b}$ and data respresent the mean + s.d. of three independent experiments. ${ }^{\star} P<0.05, t$-test. (d) Effect of FAT1 knockdown on podocyte migration. Differentiated cultured human podocytes transfected with FAT1 siRNA exhibited decreased migration (red line) compared with scrambled siRNA controls (black line). Decreased podocyte migration due to FAT1 knockdown was partially rescued by RAC/CDC42 activator II (green line). Each cell index value corresponds to the average of more than triplicates and s.d. is in only one direction for clarity. (e) Bar graphs represent the area under curves of $\mathbf{d}$ and data respresent the mean + s.d. of three independent experiments. ${ }^{\star} P<0.05$, $t$-test. (f) Cell-cell adhesion assay using calcein AM demonstrated that decreased cell-cell adhesion in fibroblasts from individual A4623 compared with control fibroblasts. Data respresent the mean \pm s.d. of more than five independent experiments in $\mathbf{f}$ and $\mathbf{g}$. ${ }^{\star} P<0.05$; $t$-test. (g) Knockdown of FAT1 by two different siRNAs in differentiated podocytes resulted in decreased cell-cell adhesion in the calcein AM assay.

Fat1 loss-of-function on glomerular integrity in vivo. Loss of Fat1 in a constitutive Fat $1^{-/-}$mouse model was previously described to cause perinatal death, features of NS with podocyte foot process effacement and brain developmental defects ${ }^{39}$. Thus, we generated a mouse model of podocyte-specific loss of Fat1 function, in which loss of Fat1 is driven by the podocyte NPHS2/podocin promoter (Pod-Cre) (Supplementary Fig. 5a,b) ${ }^{44}$.
Podocyte-specific Fat1 mutants were viable, born in ratios suggesting no embryonic lethality ( $n=11$ litters, 120 pups). Mice had no external malformation phenotype and survived to adulthood (up to 8 months). Newborn podocyte-specific Fat $1^{-/}$mice had normal renal histology, but transmission electron microscopy (TEM) revealed persistence of cuboidal podocytes, wide foot processes and tight-junction-like cell 
junctions in lieu of slit-diaphragms (Supplementary Fig. 5c-k), confirming that Fat1 is required for normal podocyte foot process and slit-diaphragm development. Adult podocyte-specific Fat1 ${ }^{-/-}$mutants developed progressive proteinuria with massive albuminuria at 4 months of age, $\sim 60$-fold higher than control mice, whereas heterozygous Fat1 mutants had only very mild albuminuria (Fig. 3a,b). However, serum albumin was not significantly different in Fat $1^{-1-}$ and control mice $(3.2 \pm 0.23$ versus $\left.3.3 \pm 0.15 \mathrm{~g} \mathrm{dl}^{-1}\right)$. Histologic examination in homozygous podocyte-specific $\mathrm{Fat1}^{-/-}$mutants revealed FSGS, with the presence of protein casts and tubulointerstitial nephropathy, while only mild mesangial expansion was observed in podocyteFat1 heterozygous mutants (Fig. 3c-f). On ultrastructural examination by TEM control, Pod-Cre and Fat $1^{f / f}$ single transgenic mice showed normal histology and ultrastructure (Fig. 3g,h,j), whereas homozygous Fat1 ${ }^{-9}$ mutants exhibited widespread foot process effacement, microvillar transformation and collapsed F-actin (Fig. 3i,k). Slit-diaphragms are remarkably decreased in Fat ${ }^{-/-}$mutants and cell junctions of effaced foot processes resemble tight junctions (Fig. 3k).

Loss of Fat1 causes CDC42-mediated renal tubular defects. To examine mechanisms of tubular defects of renal tubule architecture, we performed transient Fat1 knockdown in mouse inner medullary collecting duct (IMCD3) cells, a renal tubule cell line that has broadly been used to study tubular defects ${ }^{35,45}$. We found that knockdown of Fat1 perturbed lumen formation of IMCD3 spheroids (Fig. 4a,b), suggesting that FAT1 is involved in the regulation of apicobasal polarity. However, this lumen formation did not result from ciliogenesis because cells with FAT1 knockdown did not have a problem in ciliation (Fig. 4a). Similarly, fibroblasts from A4623 with a homozygous truncating mutation did not show any defect in number or structure of cilia compared with control fibroblasts (Supplementary Fig. 6), suggesting that FAT1 is not directly involved in ciliogenesis.

Because fibroblasts of individuals with FAT1 mutation had cell migration defects (Fig. 2b), and because Fat1 also regulates cell migration in many different cell types including podocytes, we examined whether Fat1 also regulates cell migration in IMCD3 renal tubular cells. We found that knockdown of Fat1 using two different short hairpin RNAs (shRNAs) did in fact decrease cell migration rate in IMCD3 cells (Fig. 4b,c and Supplementary Fig. 7a).

Apicobasal cell polarity is controlled by the PCP pathway and FAT family proteins are involved in this pathway ${ }^{46}$. FAT family proteins are upstream PCP proteins, Scribble is one of the PCP core proteins and RHO GTPases are downstream PCP effector proteins ${ }^{47}$. Therefore, we hypothesized that the observed defects in both lumen formation and cell migration may be mediated by a
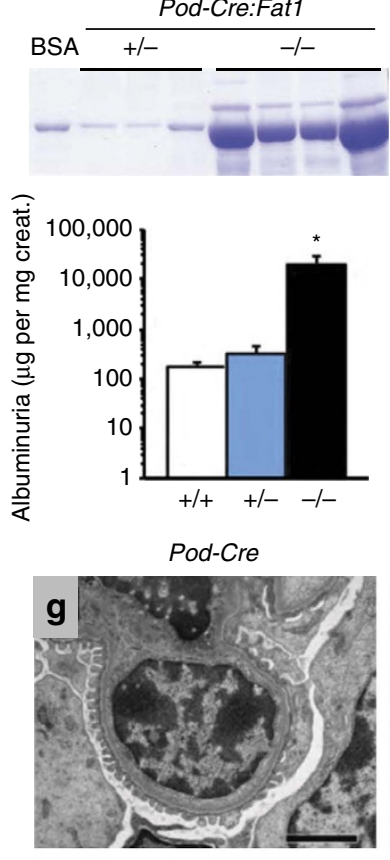
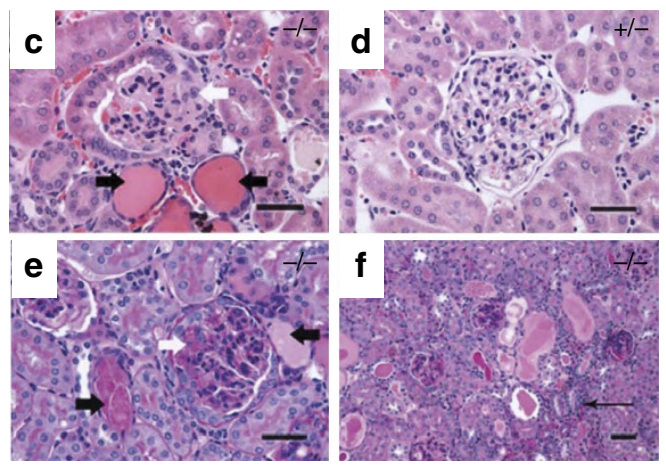

Fat1 $^{\text {f/t }}$
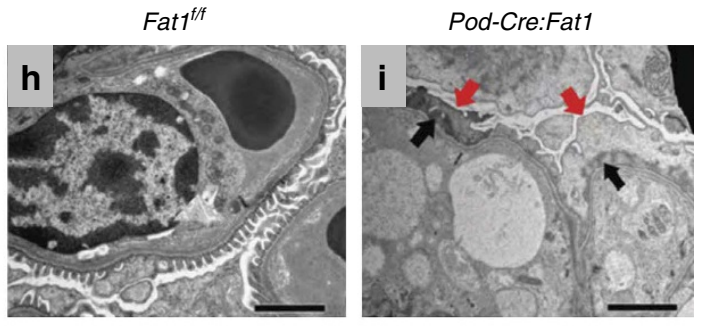
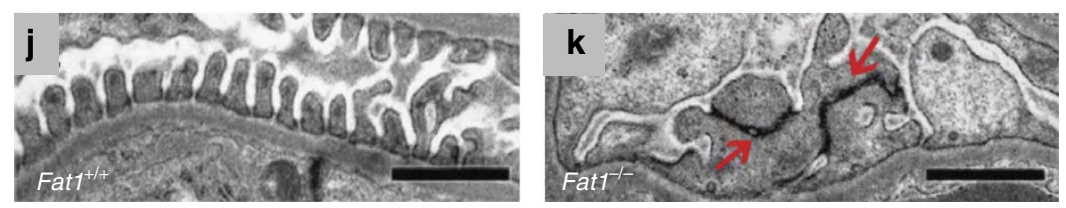

Figure 3 | Podocyte-specific Fat1 deletion causes massive proteinuria and FSGS in adult mice. (a) Urine ( $5 \mu$ l) resolved by SDS-PAGE and stained with Coomassie blue shows massive albuminuria in podocyte-Fat ${ }^{-/-}$mutants as quantitated in (b) BSA $=1 \mu \mathrm{g}$ bovine serum albumin. The number of mice studies in a-c were: $n=10\left(+/+, 5\right.$ Pod-Cre and 5 Fat $\left.7^{f / f}\right) ; n=4\left(\right.$ Pod-Cre:Fat $\left.{ }^{+/}{ }^{-}\right) ; n=5$ (Pod-Cre:Fat $\left.1^{-} /{ }^{-}\right)$. The experimental groups were compared by analysis of variance. ${ }^{\star} P<0.05, t$-test. (c) On H\&E staining, Fat ${ }^{-/-}$show focal glomerular sclerosis (white arrow) and protein casts (black arrows) compared with Fat1 ${ }^{+/-}$(d). (e,f) On PAS staining, Fat1 ${ }^{-/-}$kidneys show focal glomerular sclerosis (white arrows), protein casts (black arrows) and interstitial infiltrates (thin arrow). (c-f) Scale bars, $40 \mu \mathrm{m}$. (g-h) On TEM, Pod-Cre and Fat $\mathrm{f}^{\mathrm{f} / \mathrm{f}}$ single transgenic kidneys show normal glomerular filtration barrier ultrastructure. (i) Fat1-/- kidneys exhibit complete foot process effacement (red arrows), absence of slit-diaphragms and F-actin collapse in podocytes (black arrows). GBM and endothelium are intact. (j) Fat1 ${ }^{+/+}$kidneys show normal glomerular filtration barrier ultrastructure; (k) In Pod-Cre:Fat1 ${ }^{-/-}$, glomeruli tight junctions (red arrows) link the effaced foot processes. Scale bars, $2 \mu \mathrm{m}$ (g-i); and $1 \mu \mathrm{m}$ (h-k). 
a

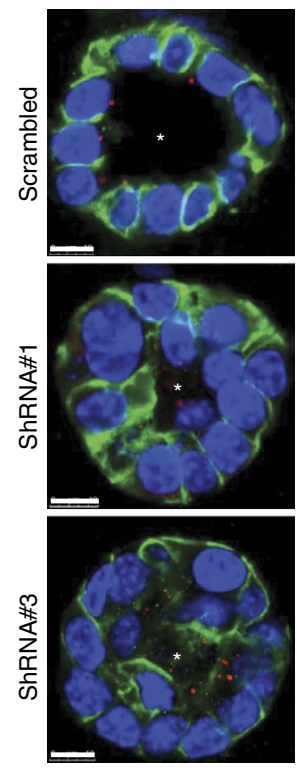

e

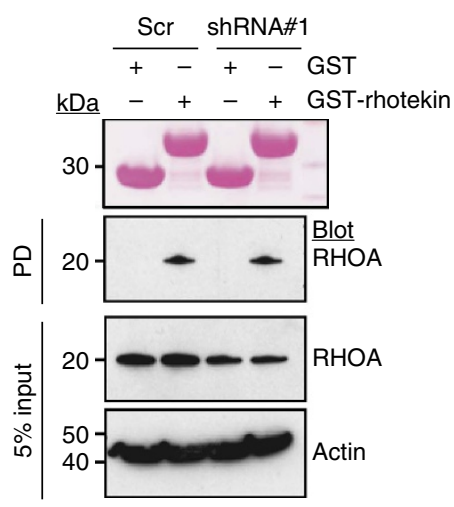

d b

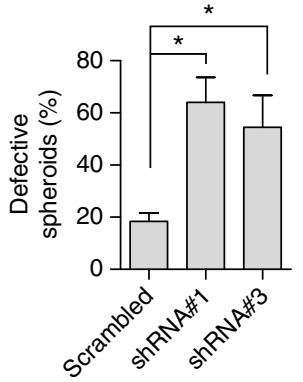

C

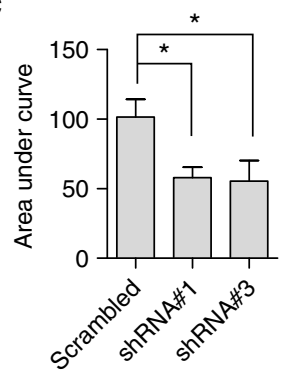

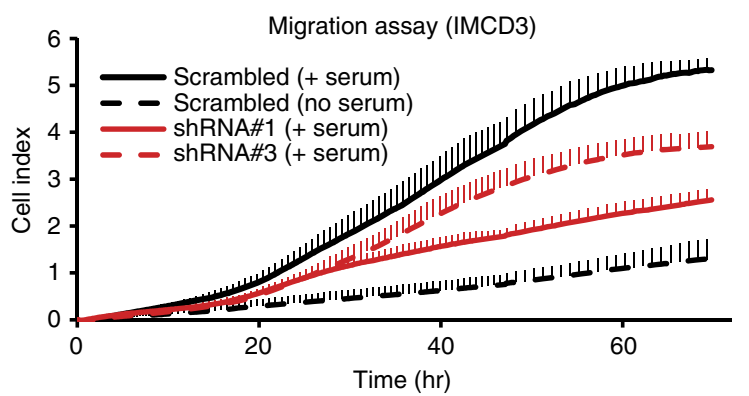

f

g
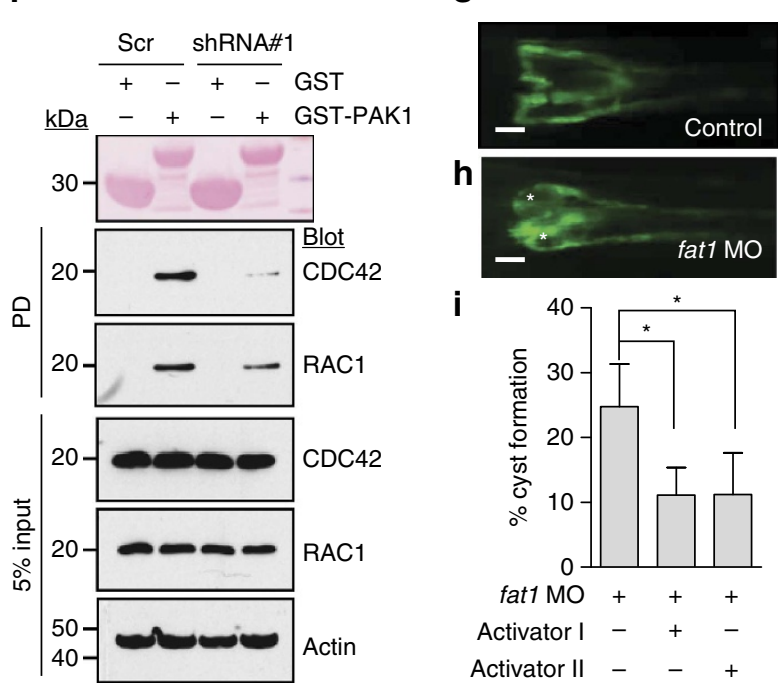

Figure 4 | Loss of FAT1 causes a renal tubular phenotype via defective RHO GTPase signalling. (a) IMCD3 cells ciliated apically and formed a spheroid containing a central lumen (asterisk) when grown in 3D matrigel culture for 3 days; lumens were perturbed on Fat1 knockdown (shRNA\#1 and shRNA\#3). Cells were stained for acetylated $\alpha$-tubulin (red), $\beta$-catenin (green) and DAPI (blue). Scale bars, $10 \mu \mathrm{m}$. (b) Percentage of abnormal spheroids. More than 50 spheroids were examined in each experiment. Data represent the mean + s.d. of three independent experiments in $\mathbf{b}, \mathbf{c}$. ${ }^{\star} P<0.05, t$-test. (c) The effect of Fat1 knockdown on cell migration. Bar graphs represent the area under curves of $\mathbf{d}$. (d) The effect of Fat7 knockdown on cell migration. Compared with baseline (dashed black line), addition of serum strongly increases migration rate in IMCD3 cells with scrambled shRNA (Scr) (solid black line). In contrast, IMCD3 cells stably transfected with Fat1 shRNAs \#1 (red continuous line) or \#3 (red dashed line) exhibited slower rate of migration. Each cell index value corresponds to the average of more than triplicates and s.d. is in only one direction for clarity. (e) Active GTP-bound RHOA precipitated from IMCD3. Cells transfected with scrambled control siRNA versus Fat1 shRNA exhibited no significant difference in relative RHOA activity. This is representative of three experiments. (f) Active GTP-bound CDC42 or RAC1 using a GST-PAK1 (CRIB) pull-down assay. Note that Fat1 knockdown leads to a significant decrease in relative CDC42 and RAC1 (31\% and 44\%, respectively) compared with Scr control cells. This is representative of four experiments. Quantification of e and $\mathbf{f}$ is presented in Supplementary Fig. 7. (g-h) fat1 morpholino-oligonucelotide (MO) was injected to Wt1b::GFP transgenic zebrafish. Zebrafish injected with control MO did not produce any phenotype. Depletion of fat 1 by a fat1 MO targeting the translation initiation site of zebrafish fat 1 caused pronephric cysts (asterisks) in 78 of 325 zebrafish embryos (24\%). Scale bars, $100 \mu \mathrm{m}$. (i) Activator I (Rho/Rac/Cdc42 activator I) reduced cyst formation to $9.7 \%$ (26 of 268 embryos), and activator II (Rac/Cdc42 activator II) to $12.0 \%$ (59 of 490 embryos). ${ }^{\star} P<0.001, \chi^{2}$-test.

disruption of RHO/RAC/CDC42 signalling. When measuring active RHOA by a GST-Rhotekin pull-down assay in IMCD3 cells, we found that on knockdown of Fatlactive GTP-bound RHOA was unaltered (Fig. 4e and Supplementary Fig. 7b). In contrast, when measuring active CDC42 and RAC 1 by GST-PAK1 pull-down assay, we found that on knockdown of
Fat1 active CDC42 and RAC1 were both decreased (Fig. 4f and Supplementary Fig. 7c,d). These results in IMCD3 cells are congruent with those in cultured podocytes (Supplementary Fig. 3) and indicate that CDC42 and RAC1 are more relevant than RHOA for the pathogenesis of the glomerulotubular disease that is caused by loss of FAT1. 
We then studied in vivo the effects on renal tubule cell architecture that we had observed in cell-based systems in vitro. We chose zebrafish as a model organism, because zebrafish embryo is amenable to genetic manipulation such as injection of morpholino (MO) and knockdown of fat1 in zebrafish is known to cause pronephric cysts ${ }^{47}$. We performed MO knockdown of fat1 in Wt1b::GFP transgenic zebrafish, in which pronephric kidney cysts can readily be visualized. We found that, whereas zebrafish injected with control MO did not produce any phenotype (Fig. 4e), depletion of fat 1 caused pronephric cysts in $24 \%$ of zebrafish embryos (Fig. $4 \mathrm{f}$ ). To further corroborate the pathogenic role of RAC1/CDC42 signalling, we tested the effect of two different $\mathrm{RHO} / \mathrm{RAC} / \mathrm{CDC} 42$ activators on rescue of the zebrafish pronephric renal cystic phenotype. We found that the chemically distinct Rho GTPase activators I and II reduced cyst formation from $31 \%$ to $9.7 \%$ and $12.0 \%$, respectively (Fig. $4 \mathrm{i}$ ).

\section{Discussion}

We here identify recessive mutations of FAT1 as causing a glomerulotubular nephropathy. Four families with FAT1 mutations presented with a combination of SRNS, tubular ectasia, haematuria and facultative neurological involvement. Mechanistically, our studies implicate RHO-like small GTPase signalling in the pathogenesis of both, the glomerular and the tubular phenotypes.

In a global Fat1 knockout mice, Fat1 null pups died within $48 \mathrm{~h}$ of birth, which was attributed to abnormal podocyte foot process fusion with obliteration of the slit membranes ${ }^{39}$. These findings are consistent with the concept that FAT1 provides spacing between cells in the kidney and plays a role in the formation of intercellular junctions in the kidney ${ }^{60}$. While our model of podocyte-specific Fat1 deletion caused identical podocyte foot process fusion at birth, we did not observe perinatal lethality, suggesting that perinatal lethality with global Fat1 loss reflects a different developmental problem.

Fat1 null mice also exhibited partially penetrant midline developmental defects including holoprosencephaly, as expected from strong Fat1 expression within the neuroepithelium. ${ }^{39}$ This mouse model is consistent with our finding of a combined renal and neurologic disease phenotype in human recessive FAT1 mutation. Our observation of incomplete penetrance of the neuronal involvement in individuals with FAT1 mutation parallels the findings in the mouse model and is most likely due to compensation by the other FAT proteins (FAT2, 3 or 4$)^{38}$, and to the fact that all of the individuals with two recessive FAT1 mutations (except A4623) carried at least one hypomorphic allele, that is, an allele that is expected not to convey full loss-of-function (Table 1). It is also consistent with the finding that individual A4623, who carries the homozygous truncating mutation, has a disease phenotype with the most severe neuronal involvement (Table 1).

Cooperation of different members of FAT cadherins was demonstrated in mouse models of combined loss-of-function of Fat4 and Fat1, where FAT proteins cooperate in regulating multiple aspects of tissue morphogenesis in the kidney and other organs. Fat function affected renal tubular elongation, neural tube formation and cochlear morphology via its effects on mechanisms of PCP. In this context, removal of one copy of the murine Fat1 gene exacerbated the renal tubular cyst phenotype of mice ${ }^{48}$.

Taken together, our cell-based and in vivo studies using mouse and zebrafish suggest that the glomerular SRNS-like disease phenotype results from impaired cell migration of glomerular podocytes. Indeed, defective podocyte migration is a disease mechanism common to multiple different monogenic forms of $\mathrm{SRNS}^{30}$. Likewise, defective cell migration was recently recognized as a central mechanism of renal tubular regeneration ${ }^{49}$. Interestingly, Fat1 knockdown limited migration of renal tubule cells and also impaired their ability to form lumens in cultured spheroids, suggesting that also the renal tubular defect may be mediated by impaired cell migration.

We further demonstrated that RAC1 and CDC42 activity are impaired in both cultured podocytes and tubular cells with decreased Fat1 expression, and that activation of these small GTPases partially corrects defective cellular migration that occur with Fat1 deficiency. This suggests that the pathogenesis of both the renal glomerular and tubular defects is mediated by decreased RAC1/CDC42 as a potential unifying mechanism underlying both the glomerular and tubular disease phenotypes. We confirm the relevance of this disease mechanism by showing that two different RAC1/CDC42 activators mitigate the pronephric cyst phenotype in zebrafish.

Data from human genetics and animal models have shown the importance of RHO GTPase signalling in CKDs ${ }^{15,16,50-52}$. ARHGAP24 or ARHGDIA mutations identified in human SRNS cause upregulation of both RAC1 and CDC42 and lead to increased podocyte migration ${ }^{15,16}$. This is congruent with the finding that active RAC1 (but not RHOA) is upregulated in Arhgdia ${ }^{-1-}$ mice. However, CDC42 activity has not been examined in the Arhgdia ${ }^{-1-}$ mouse model ${ }^{53}$. Scott et al. ${ }^{50}$ showed that podocyte-specific deletion of CDC42 (but not RHOA and RAC1) in mice results in foot process effacement and proteinuria. INF2 mutations also decrease active CDC42 at the plasma membrane and cause mislocalization of CDC42 within the cytoplasm ${ }^{52}$. Therefore, either aberrant activation or inactivation of CDC42 interferes with podocyte function. In addition, $c d c 42$ knockdown in zebrafish led to hydrocephalus, body oedema and pronephric cysts, and kidney tubule-specific deletion of CDC42 resulted in renal cysts and renal failure within weeks of birth ${ }^{51}$, indicating that CDC42 is also important for proper tubular function. In this study, we show that the impaired podocyte migration and the pronephric cysts in fat1-knockdown zebrafish were partially rescued by a RAC/CDC42 activator, suggesting that the combined glomerulotubular phenotype resulting from FAT1 mutations is mediated by decreased RAC1 and CDC42 in both glomeruli and tubules.

Recently Morris et al. ${ }^{43}$ identified FAT1 mutations in human cancers including glioblastoma and colorectal cancer, and showed that loss of FAT1 leads to aberrant activation of Wnt signalling. The FAT1 mutations identified by Morris et al. ${ }^{43}$ were somatic and mostly heterozygous. However, in the glomerulotubular disease described here renal disease resulting from FAT1 mutations is recessive, requiring two mutant alleles, which are inherited from the heterozygous parents. It is not clear whether individuals with recessive FAT1 mutations will develop cancer later in life. However, it is important to note that two individuals (A3027 and A789) had Ewing sarcoma and Hodgkin's lymphoma, respectively (Table 1). How the respective FAT1 mutations identified in these individuals might contribute to neoplastic disease will require further focused study.

FAT1 is implicated in Hippo signalling through the interaction with Scribble and Hippo signalling is required for normal pronephros development in zebrafish ${ }^{47}$. Yes-associated protein (Yap) is a downstream transcriptional effector of Hippo signalling and is essential for nephron induction and morphogenesis 54 . Recently, it was shown that Cdc42 acts upstream of Yap during development to promote Yap-dependent gene expression and shape functioning nephrons ${ }^{54}$. Therefore, it is also conceivable that the glomerulotubular nephropathy caused by loss of FAT1 may result from defective Hippo signalling due to decreased Cdc42 activity, which we have shown in this study (Fig. $4 \mathrm{f}$ and Supplementary Fig. 3a). 
Because SRNS cause $\sim 15 \%$ of all CKD in the first two decades of life, the balance of RAC1/CDC42 signalling may be a worthwhile target when developing drugs to prevent ESKD that is caused by SRNS.

\begin{abstract}
Methods
Study participants. Following informed consent for WES, we obtained clinical data and blood samples from individuals with SRNS or NPHP from worldwide sources. Approval for human subjects' research was obtained from the University of Michigan and Boston Children's Hospital Institutional Review Boards and from relevant local Review Boards. Deposition of WES data in public databases was not obtained at the time. The diagnosis of NS or NPHP was made by (paediatric) nephrologists based on standardized clinical and renal histologic criteria ${ }^{55}$. Renal biopsies were evaluated by renal pathologists. Clinical data were obtained using a standardized questionnaire (http://www.renalgenes.org).
\end{abstract}

Whole exome sequencing. We combined WES with $\mathrm{HM}$ as established previously ${ }^{32,36}$. For $\mathrm{HM}^{32}$ the 'Human Mapping $250 \mathrm{k}$ StyI' array (Affymetrix) was utilized. Genomic DNA samples were hybridized, and scanned using the manufacturer's standard protocol at the University of Michigan Core Facility (www.michiganmicroarray.com). Non-parametric logarithm of odds (LOD) scores were calculated using a modified version of the program GENEHUNTER 2.1 (refs $56,57)$ through stepwise use of a sliding window with sets of 110 SNPs using the program ALLEGRO ${ }^{58}$. Genetic regions of homozygosity by descent ('homozygosity peaks') were plotted across the genome as candidate regions for recessive diseasecausing genes as described ${ }^{32,35}$. Disease allele frequency was set at 0.0001 , and Caucasian marker allele frequencies were used. Variant burden analysis was performed as previously described ${ }^{59}$ using Agilent SureSelect human exome capture arrays (Life technologies) with next-generation sequencing on an Illumina sequencing platform. Sequence reads were mapped against the human reference genome (NCBI build 37/hg19) using CLC Genomics Workbench (version 6.5.1) software (CLC bio, Aarhus, Denmark). Mutation calling (Supplementary Table 1) was performed by geneticists/cell biologists, who have knowledge of clinical phenotypes, pedigree structure, HM and WES evaluation. All the coding exons of FAT1 were examined by applying a high-throughput mutation analysis method of microfluidic array-based muyltiplex PCR (Fluidigm 48.48 Access Array) and consecutive barcoded next-generation sequencing (MiSeq, Illumina) ${ }^{37}$.

Zebrafish maintenance and injections. Approval for zebrafish research was obtained from the University Committee on the Use and Care of Animals (UCUCA) of the University of Freiburg. Transgenic wt 1b::GFP zebrafish line was raised and maintained as described ${ }^{60}$. Fertilized eggs were microinjected with $4 \mathrm{nl}$ of injection solution at the one-to-two-cell stage with MOs (Gene Tools LLC) diluted in $200 \mathrm{mM} \mathrm{KCl}, 0.1 \%$ Phenol Red (Sigma-Aldrich Corporation) and $10 \mathrm{mM}$ HEPES. $z$ Fat $1 \mathrm{MO}$ has been previously described ${ }^{47}$. About 0.5 pmol of zebrafish p53 MO (5'-GCGCCATTGCTTTGCAAGAATTG-3', Gene Tools) was always co-injected to reduce the unspecific effects of the $\mathrm{MOs}^{61}$. About $1.6 \mathrm{pmol} z \mathrm{Fat} 1 \mathrm{MO}$ was injected either alone or together with either $1 \mu \mathrm{g} \mathrm{ml}^{-1}$ of Rho/Rac/Cdc42 Activator I or $0.5 \mathrm{U} \mathrm{ml}^{-1}$ of Rac/Cdc42 activator II (Cytoskeleton). Scoring of percentage of cyst formation and zebrafish embryo imaging was done on mixed male and female embryos at $48 \mathrm{~h}$ post fertilization under a Leica MZ16 stereo microscope (Leica).

Podocyte conditional Fat1-deficient mice. To develop the Fat1 conditional allele in mice, homologous recombination was used to introduce LoxP sites flanking exon 2. The targeting construct for the Fat1 locus was generated from a C57/BL6 strain bacterial artificial chromosome (BAC) clone (Children's Hospital Oakland Research Institute) by homologous recombination BAC engineering techniques (Open Biosystems). All junctions and overall structure were confirmed by sequencing and long-range PCR. LoxP sites were introduced on each side of exon 2, which encodes the signal peptide and $\mathrm{N}$-terminal 1,089 aa of Fat1. The neomycin selection cassette was flanked by Frt sequences for removal by Flp recombinase.

Electroporation of the linearized construct into WW6 embryonic stem (ES) cells, followed by selection with G418 and ganciclovir, yielded a high percentage $(\sim 60 \%)$ of homologous recombinants. A Fat1 ${ }^{+/ l o x}$ ES clone injected into C57/BL6 blastocysts yielded three highly chimeric mice that transmitted the targeted allele to offspring. The Frt-flanked neo cassette was removed in a cross with a FLPeR mouse

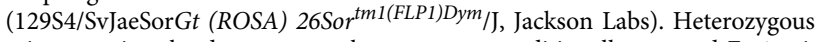
mice were interbred to generate homozygous conditionally targeted Fatl mice $\left(\right.$ Fat $\left.1^{\text {loxP/loxP }}\right)$ in a mixed 129SV/BL6 genetic background. To delete fat 1 selectively in podocytes, female Fat $1^{+/ f l}$ mice were bred with male podocin-cre mice ${ }^{44}$ and double heterozygous were backcrossed to obtain pod-cre:Fat $1^{\text {fllfl }}$. Podocin-cre mice were provided by Dr Holzman. All animals were housed in pathogen-free conditions, and protocols approved by the standing committees of the Albert Einstein Institute for Animal Studies and the Yale Animal Resources Center. Urinary albumin was measured using mouse albumin ELISA (Bethyl Laboratories) and SDS-PAGE/Coomassie Blue staining as previously described ${ }^{62}$. Plasma and
$24 \mathrm{~h}$ urine creatinine were measured by HPLC ${ }^{62}$. Renal morphology was examined in newborn (1-3-day-old) mice and in 4-8-month-old mice by light microscopy (haematoxylin and eosin- and periodic acid-Schiff-stained sections) and by TEM using standard techniques ${ }^{62}$.

Cell culture and transfection. The immortalized human podocytes ${ }^{63}$ were kindly provided by Dr Saleem (the University of Bristol) and maintained at the permissive temperature of $33^{\circ} \mathrm{C}$ in RPMI + GlutaMAX-I (Gibco) supplemented with $10 \%$ fetal bovine serum, penicillin $\left(50 \mathrm{IU} \mathrm{ml}^{-1}\right) /$ streptomycin $\left(50 \mu \mathrm{g} \mathrm{ml}^{-1}\right)$ and insulin-transferrin-selenium-X. To differentiate podocytes, they were cultured at $37^{\circ} \mathrm{C}$ for 14 days. The FAT1-specific and control scrambled short interfering RNAs (siRNAs) were purchased from Dharmacon. siRNAs were transfected into podocytes using Lipofectamine RNAiMAX (Invitrogen). Mouse kidney collecting duct IMCD3 cells were obtained from the ATCC (CRL-2123) and cultured in DMEM/F12 with $10 \%$ fetal bovine serum and penicillin $\left(50 \mathrm{IU} \mathrm{ml}^{-1}\right) /$ streptomycin $\left(50 \mu \mathrm{g} \mathrm{ml}^{-1}\right)$. To achieve stable knockdown of FAT1, IMCD3 cells were transducted with lentivirus, which contained shRNAs. The target sequences of siRNAs and shRNAs used in this study are in Supplementary Table 2. The shRNAstable IMCD3 cells were selected and maintained with $8 \mu \mathrm{g} \mathrm{ml}^{-1}$ puromycin. Human fibroblasts were grown in DMEM supplemented with 15\% FBS, penicillin $\left(50 \mathrm{IU} \mathrm{ml}^{-1}\right) /$ streptomycin $\left(50 \mu \mathrm{g} \mathrm{ml}^{-1}\right)$ and non-essential amino acids (Invitrogen). Rho/Rac/Cdc42 activator I and Rac/Cdc42 activator II were purchased from the Cytoskeleton, Inc.

Lentiviral overexpression. The coding sequence of CDC42-T17N was subcloned to pLenti-CMV-Blast vector (Addgene plasmid $\# 17451)^{64}$. For overexpression in differentiated cultured podocytes, HEK293T cells were transfected with pLentiCMV-Blast-CDC42-T17N and lentiviral packaging vectors, pRSV-Rev, pMDLg/ pRRE and pMD2.G. The lentivirus-contacting medium was collected after $48 \mathrm{~h}$ and podocytes were infected on day 12 of differentiation with $8 \mu \mathrm{g} \mathrm{ml}^{-1}$ polybrene-containing virus media. Podocytes were used for migration assay for $72 \mathrm{~h}$ after infection.

Immunoblotting and GST pull-down assay. These experiments were performed as described previously ${ }^{65}$. Anti-acetylated- $\alpha$-tubulin (acetyal K40), Anti- $\beta$-actin (AC-15) (Abcam), anti-Rac1 (102/Rac1), anti-Cdc42 (44/CDC42), anti- $\beta$-catenin (14/Beta-Catenin) (BD Transduction Laboratories), anti-RhoA (26C4) (Santa Cruz Biotechnology) and CEP164 (13) (Sigma-Aldrich) were purchased from commercial sources. The FAT1-GST antibody, which was generated against C-terminal 385 aa of mouse FAT1, was previously described by Moeller et al ${ }^{40}$. All of the antibodies were used at 1:1,000 for immunoblotting. Uncropped scans of the western blots are shown in Supplementary Fig. 8.

Migration assay. Real-time migration assay was performed using the xCELLigence system (ACEA Biosciences) in CIM-plate 16 according to the manufacturer's instruction. Briefly, $4 \times 10^{4}$ cells of fibroblasts, cultured human podocytes or IMCD3 were plated in serum-free media in the upper chamber. The lower chambers were with $10 \%$ FBS for chemoattraction or with serum-free media. The obtained data were analysed using the RTCA software. Results are presented as the time versus cell index curve.

Spheroid assay. IMCD3 cells were trypsinized and resuspended cells were then mixed 1:1 with growth factor-depleted matrigel (BD Bioscience). This mixture was then moved to the well of a Nunc Lab-Tek II chambered coverglass (Thermo Fisher) and allowed to polymerize ( $45 \mathrm{~min}$ to $1 \mathrm{~h}$ ) at $37^{\circ} \mathrm{C}$. Once the matrigel was fully solidified, warm medium was added drop-wise to the matrix until covered. After $72 \mathrm{~h}$, the cells had formed spheroids with visible cleared lumens. The matrigels were then fixed in fresh 4\% PFA for $30 \mathrm{~min}$ at room temperature and subsequently permeabilized for $15 \mathrm{~min}$ in gelatin-dissolving PBS and $0.5 \%$ Triton $\mathrm{X}-100$. The primary antibodies (Anti-acetylated- $\alpha$-tubulin 1:2,000 and $\beta$-catenin, 1:500) were incubated at $4{ }^{\circ} \mathrm{C}$ overnight. Fluorescent images were obtained with a Leica SP5X laser scanning microscope.

Fluorescence-based cell-cell adhesion assay. This assay was performed as described previously ${ }^{43}$. Fibroblasts or cultured human podocytes were resuspended at $1 \times 10^{6}$ cells per $\mathrm{ml}$ in serum-free medium supplemented with $5 \mu \mathrm{M}$ calcein $\mathrm{AM}$ and incubated at $37^{\circ} \mathrm{C}$ for $30 \mathrm{~min}$. Cells were washed twice with serum-free medium, and $1 \times 10^{5}$ cells were added to microplate wells containing confluent (unlabelled) cells. Calcein-labelled cells were allowed to adhere for $45 \mathrm{~min}$ at $37^{\circ} \mathrm{C}$. Non-adherent calcein-labelled cells were washed away with medium, and PBS was added to each well. Fluorescence was measured at an absorbance of $494 \mathrm{~nm}$ and emission of $517 \mathrm{~nm}$ using a SpectraMax Multilabel Microplate Reader (Molecular Devices).

\section{References}

1. Smith, J. M., Stablein, D. M., Munoz, R., Hebert, D. \& McDonald, R. A. Contributions of the transplant registry: The 2006 Annual Report of the North 
American Pediatric Renal Trials and Collaborative Studies (NAPRTCS). Pediatr. Transplant. 11, 366-373 (2007).

2. Ruf, R. G. et al. Patients with mutations in NPHS2 (podocin) do not respond to standard steroid treatment of nephrotic syndrome. J. Am. Soc. Nephrol. 15, 722-732 (2004).

3. Hildebrandt, F. \& Heeringa, S. F. Specific podocin mutations determine age of onset of nephrotic syndrome all the way into adult life. Kidney Int. 75, 669-671 (2009).

4. Somlo, S. \& Mundel, P. Getting a foothold in nephrotic syndrome. Nat. Genet. 24, 333-335 (2000).

5. Tryggvason, K., Patrakka, J. \& Wartiovaara, J. Hereditary proteinuria syndromes and mechanisms of proteinuria. N. Engl. J. Med. 354, 1387-1401 (2006)

6. Sadowski, C. E. et al. A single-gene cause in $29.5 \%$ of cases of steroid-resistant nephrotic syndrome. J. Am. Soc. Nephrol. 26, 1279-1289 (2015).

7. Kestila, M. et al. Positionally cloned gene for a novel glomerular protein--nephrin--is mutated in congenital nephrotic syndrome. Mol. Cell 1, 575-582 (1998).

8. Boute, N. et al. NPHS2, encoding the glomerular protein podocin, is mutated in autosomal recessive steroid-resistant nephrotic syndrome. Nat. Genet. 24, 349-354 (2000)

9. Shih, N. Y. et al. Congenital nephrotic syndrome in mice lacking CD2-associated protein. Science 286, 312-315 (1999).

10. Hinkes, B. et al. Positional cloning uncovers mutations in PLCE1 responsible for a nephrotic syndrome variant that may be reversible. Nat. Genet. 38, 1397-1405 (2006).

11. Kaplan, J. M. et al. Mutations in ACTN4, encoding alpha-actinin-4, cause familial focal segmental glomerulosclerosis. Nat. Genet. 24, 251-256 (2000).

12. Brown, E. J. et al. Mutations in the formin gene INF2 cause focal segmental glomerulosclerosis. Nat. Genet. 42, 72-76.

13. Mele, C. et al. MYO1E mutations and childhood familial focal segmental glomerulosclerosis. N. Engl. J. Med. 365, 295-306 (2011).

14. Gbadegesin, R. A. et al. Mutations in the gene that encodes the F-actin binding protein anillin cause FSGS. J. Am. Soc. Nephrol. 25, 1991-2002 (2014).

15. Akilesh, S. et al. Arhgap24 inactivates Rac1 in mouse podocytes, and a mutant form is associated with familial focal segmental glomerulosclerosis. J. Clin. Invest. 121, 4127-4137 (2011).

16. Gee, H. Y. et al. ARHGDIA mutations cause nephrotic syndrome via defective RHO GTPase signalling. J. Clin. Invest. 123, 3243-3253 (2013).

17. Gee, H. Y. et al. KANK deficiency leads to podocyte dysfunction and nephrotic syndrome. J. Clin. Invest. 125, 2375-2384 (2015).

18. Has, C. et al. Integrin alpha3 mutations with kidney, lung, and skin disease. $N$. Engl. J. Med. 366, 1508-1514 (2012).

19. Hasselbacher, K. et al. Recessive missense mutations in LAMB2 expand the clinical spectrum of LAMB2-associated disorders. Kidney Int. 70, 1008-1012 (2006).

20. Zenker, M. et al. Human laminin beta2 deficiency causes congenital nephrosis with mesangial sclerosis and distinct eye abnormalities. Hum. Mol. Genet. 13, 2625-2632 (2004).

21. Antignac, C. Molecular basis of steroid-resistant nephrotic syndrome. Nefrologia 25(Suppl 2): 25-28 (2005).

22. Ashraf, S. et al. ADCK4 mutations promote steroid-resistant nephrotic syndrome through CoQ10 biosynthesis disruption. J. Clin. Invest. 123, 5179-5189 (2013)

23. Heeringa, S. F. et al. COQ6 mutations in human patients produce nephrotic syndrome with sensorineural deafness. J. Clin. Invest. 121, 2013-2024 (2011).

24. Mollet, J. et al. Prenyldiphosphate synthase, subunit 1 (PDSS1) and $\mathrm{OH}$ benzoate polyprenyltransferase (COQ2) mutations in ubiquinone deficiency and oxidative phosphorylation disorders. J. Clin. Invest. 117, 765-772 (2007).

25. Diomedi-Camassei, F. et al. COQ2 nephropathy: a newly described inherited mitochondriopathy with primary renal involvement. J. Am. Soc. Nephrol. 18, 2773-2780 (2007)

26. Yang, Y. et al. and PAX-2 podocyte expression in Denys-Drash syndrome and isolated diffuse mesangial sclerosis. Am. J. Pathol. 154, 181-192 (1999).

27. Reiser, J. et al. TRPC6 is a glomerular slit diaphragm-associated channel required for normal renal function. Nat. Genet. 37, 739-744 (2005).

28. Winn, M. P. et al. A mutation in the TRPC6 cation channel causes familial focal segmental glomerulosclerosis. Science 308, 1801-1804 (2005).

29. Gee, H. Y. et al. Mutations in EMP2 cause childhood-onset nephrotic syndrome. Am. J. Hum. Genet. 94, 884-890 (2014).

30. Faul, C., Asanuma, K., Yanagida-Asanuma, E., Kim, K. \& Mundel, P. Actin up: regulation of podocyte structure and function by components of the actin cytoskeleton. Trends Cell Biol. 17, 428-437 (2007).

31. Sadowski, C. E. et al. A single-gene cause in $29.5 \%$ of cases of steroid-resistant nephrotic syndrome. J. Am. Soc. Nephrol. 26, 1279-1289 (2014).

32. Hildebrandt, F. et al. A systematic approach to mapping recessive disease genes in individuals from outbred populations. PLoS Genet. 5, e1000353 (2009).

33. Matejas, V. et al. Mutations in the human laminin beta2 (LAMB2) gene and the associated phenotypic spectrum. Hum. Mutat. 31, 992-1002 (2010).
34. Ng, S. B. et al. Targeted capture and massively parallel sequencing of 12 human exomes. Nature 461, 272-276 (2009).

35. Otto, E. A. et al. Candidate exome capture identifies mutation of SDCCAG8 as the cause of a retinal-renal ciliopathy. Nat. Genet. 42, 840-850 (2010).

36. Gee, H. Y. et al. Whole-exome resequencing distinguishes cystic kidney diseases from phenocopies in renal ciliopathies. Kidney Int. 85, 880-887 (2014).

37. Halbritter, J. et al. Identification of 99 novel mutations in a worldwide cohort of 1,056 patients with a nephronophthisis-related ciliopathy. Hum. Genet. 132, 865-884 (2013).

38. Sadeqzadeh, E., de Bock, C. E. \& Thorne, R. F. Sleeping giants: emerging roles for the fat cadherins in health and disease. Med. Res. Rev. 34, 190-221 (2014).

39. Ciani, L., Patel, A., Allen, N. D. \& ffrench-Constant, C. Mice lacking the giant protocadherin mFAT1 exhibit renal slit junction abnormalities and a partially penetrant cyclopia and anophthalmia phenotype. Mol. Cell Biol. 23, 3575-3582 (2003).

40. Moeller, M. J. et al. Protocadherin FAT1 binds Ena/VASP proteins and is necessary for actin dynamics and cell polarization. EMBO J. 23, 3769-3779 (2004).

41. Mao, J. et al. Myole impairment results in actin reorganization, podocyte dysfunction, and proteinuria in zebrafish and cultured podocytes. PloS ONE $\mathbf{8}$, e72750 (2013).

42. Nishikawa, Y. et al. Human FAT1 cadherin controls cell migration and invasion of oral squamous cell carcinoma through the localization of beta-catenin. Oncol. Rep. 26, 587-592 (2011)

43. Morris, L. G. et al. Recurrent somatic mutation of FAT1 in multiple human cancers leads to aberrant Wnt activation. Nat. Genet. 45, 253-261 (2013).

44. Moeller, M. J., Sanden, S. K., Soofi, A., Wiggins, R. C. \& Holzman, L. B. Podocyte-specific expression of cre recombinase in transgenic mice. Genesis $\mathbf{3 5}$, 39-42 (2003).

45. Chaki, M. et al. Exome capture reveals ZNF423 and CEP164 mutations, linking renal ciliopathies to DNA damage response signalling. Cell 150, 533-548 (2012).

46. Hariharan, I. K. \& Bilder, D. Regulation of imaginal disc growth by tumorsuppressor genes in Drosophila. Annu. Rev. Genet. 40, 335-361 (2006).

47. Skouloudaki, K. et al. Scribble participates in Hippo signalling and is required for normal zebrafish pronephros development. Proc. Natl Acad. Sci. USA 106, 8579-8584 (2009).

48. Saburi, S. et al. Loss of Fat4 disrupts PCP signalling and oriented cell division and leads to cystic kidney disease. Nat. Genet. 40, 1010-1015 (2008).

49. Palmyre, A. et al. Collective epithelial migration drives kidney repair after acute injury. PloS ONE 9, e101304 (2014).

50. Scott, R. P. et al. Podocyte-specific loss of cdc42 leads to congenital nephropathy. J. Am. Soc. Nephrol. 23, 1149-1154 (2012).

51. Choi, S. Y. et al. Cdc42 deficiency causes ciliary abnormalities and cystic kidneys. J. Am. Soc. Nephrol. 24, 1435-1450 (2013).

52. Boyer, O. et al. INF2 mutations in Charcot-Marie-Tooth disease with glomerulopathy. N. Engl. J. Med. 365, 2377-2388 (2011)

53. Shibata, S. et al. Modification of mineralocorticoid receptor function by Rac1 GTPase: implication in proteinuric kidney disease. Nat. Med. 14, 1370-1376 (2008).

54. Reginensi, A. et al. Yap- and Cdc42-dependent nephrogenesis and morphogenesis during mouse kidney development. PLoS Genet. 9, e1003380 (2013).

55. ISKDC. Primary nephrotic syndrome in children: Clinical significance of histopathologic variants of minimal change and of diffuse mesangial hypercellularity: a report of the International Study of Kidney Disease in Children. Kidney Int. 20, 765-771 (1981).

56. Kruglyak, L., Daly, M. J., Reeve-Daly, M. P. \& Lander, E. S. Parametric and nonparametric linkage analysis: a unified multipoint approach. Am. J. Hum. Genet. 58, 1347-1363 (1996).

57. Strauch, K. et al. Parametric and nonparametric multipoint linkage analysis with imprinting and two-locus-trait models: application to mite sensitization Am. J. Hum. Genet. 66, 1945-1957 (2000).

58. Gudbjartsson, D. F., Jonasson, K., Frigge, M. L. \& Kong, A. Allegro, a new computer program for multipoint linkage analysis. Nat. Genet. 25, 12-13 (2000).

59. Boyden, L. M. et al. Mutations in kelch-like 3 and cullin 3 cause hypertension and electrolyte abnormalities. Nature 482, 98-102 (2012).

60. Perner, B., Englert, C. \& Bollig, F. The Wilms tumor genes wtla and wt1b control different steps during formation of the zebrafish pronephros. Dev. Biol. 309, 87-96 (2007).

61. Robu, M. E. et al. p53 activation by knockdown technologies. PLoS Genet. 3, e78 (2007).

62. Reidy, K. J. et al. Excess podocyte semaphorin-3A leads to glomerular disease involving plexinA1-nephrin interaction. Am. J. Pathol. 183, 1156-1168 (2013).

63. Saleem, M. A. et al. A conditionally immortalized human podocyte cell line demonstrating nephrin and podocin expression. J. Am. Soc. Nephrol. 13, 630-638 (2002). 
64. Campeau, E. et al. A versatile viral system for expression and depletion of proteins in mammalian cells. PloS ONE 4, e6529 (2009).

65. Gee, H. Y., Tang, B. L., Kim, K. H. \& Lee, M. G. Syntaxin 16 binds to cystic fibrosis transmembrane conductance regulator and regulates its membrane trafficking in epithelial cells. J. Biol. Chem. 285, 35519-35527 (2010).

\section{Acknowledgements}

We thank the families who contributed to this study, UK Renal Rare Disease Registry (www.renalradar.org) and Dr Susan Arbuckle (Children's Hospital Westmead) and Dr David Manson (Toronto) for contributing renal pathology materials and radiology materials, respectively. Sequencing of UK patients was supported by the National Institute for Health Research (NIHR) Biomedical Research Centre based at Guy's and St Thomas' NHS Foundation Trust and King's College London and the Guys and St Thomas' Hospital Charity. The views expressed are those of the author(s) and not necessarily those of the NHS, the NIHR or the Department of Health. We thank Maria Ericsson (Harvard Medical School Electron Microscopy Facility) for technical assistance. This research was supported by grants from the National Institutes of Health to F.H. (DK076683, DK068306), to E.A.O. (DK090917), to A.T. (DK059333), to N.E.S (HL104518) and by a grant from the Nephcure Foundation (to F.H.). H.Y.G. was supported by an OFD/CTREC/RRRC Career Development Fellowship, a Nephcure-ASN Foundation Kidney Research Grant and Basic Science Research Program through the National Research Foundation of Korea by the Ministry of Science, ICT \& Future planning (2015R1D1A1A01056685). G.W. was supported by grants from the Deutsche Forschungsgemeinschaft (SFB 1140), and from the European Community's Seventh Framework Programme (grant agreement 241955, SYSCILIA). F.H. is the Warren E. Grupe Professor of Pediatrics at Harvard Medical School.

\section{Author contributions}

H.Y.G., C.E.S., J.D.P., M.S., S.L., S.A., D.A.B., J.H., H.F., R.A., V.V-W., E.A.O., R.P.L. and F.H. prepared and evaluated genetic mapping and exome sequences. C.E.S., J.D.P. and M.S. identified mutations in the human FAT1 gene. H.Y.G. and K.J.C. performed cell adhesion assay, spheroid assay, GTPase studies, immunofluorescence and cell migration assay. T.A.C., L.G.T.M., C.f-C., N.A., H.M. and L.B.H. provided and evaluated critical cell lines and animal resources. P.K.A. performed all mouse studies. N.E.S.S developed the conditional Fat1 mouse model and critically evaluated mouse data. A.T. designed, analysed and wrote mouse studies. T.A.Y. and G.W. preformed zebrafish studies. R.B., H.K., M.W., A.G., T.K., D.V.M., M.S., W.T.K., S.I.A, R.P.V., C.L., J.C.T., R.B., N.A.S., M.A.S. and F.H. recruited patients and gathered detailed clinical information for the study. All authors critically reviewed the paper. F.H. conceived and directed the project and wrote the manuscript with help from H.Y.G. and A.T.

\section{Additional information}

Supplementary Information accompanies this paper at http://www.nature.com/ naturecommunications

Competing financial interests: The authors declare no competing financial interests.

Reprints and permission information is available online at http://npg.nature.com/ reprintsandpermissions/

How to cite this article: Gee, H. Y. et al. FAT1 mutations cause a glomerulotubular nephropathy. Nat. Commun. 7:10822 doi: 10.1038/ncomms10822 (2016).

\section{(c) (i)}

This work is licensed under a Creative Commons Attribution 4.0 International License. The images or other third party material in this article are included in the article's Creative Commons license, unless indicated otherwise in the credit line; if the material is not included under the Creative Commons license, users will need to obtain permission from the license holder to reproduce the material. To view a copy of this license, visit http://creativecommons.org/licenses/by/4.0/ 